\title{
Exposición a la luz nocturna, disrupción del ritmo circadiano y la obesidad
}

\section{Dr. Arturo Orrego Monsalve}

Médico Internista Endocrinólogo, Miembro Honorario de la Asociación Colombiana de Endocrinología, Diabetes y Metabolismo

Fecha de recepción: 8/06/2016

Fecha de aceptación: 30/06/2016

\section{Generalidades}

$\mathrm{L}$ a obesidad es una condición patológica caracterizada por la acumulación de exceso de tejido adiposo y está asociada con enfermedades de severidad variable como: diabetes mellitus, enfermedades cardiovasculares, hipertensión arterial, asma, cáncer y trastornos de la función reproductora ${ }^{(1)}$. Además de los riesgos tradicionales en la presentación de la obesidad con la dieta hipercalórica y la vida sedentaria, existen otros factores ambientales responsables del desarrollo de esta entidad y de su mantenimiento ${ }^{(2)}$.

En la última centuria han ocurrido profundos cambios en el estilo de vida de los humanos, avances en los viajes y las comunicaciones, modernización de las viviendas y la erradicación de varias enfermedades ${ }^{(3)}$. Un cambio ambiental que ha modificado profundamente el modo de vida del ser humano ha sido la luz eléctrica. La utilización de la iluminación eléctrica durante la noche produjo una revolución en la industria, permitiendo que se pudieran tener empleados en turnos diurnos y nocturnos y que los trabajadores pudieran escoger uno u otro periodo, según las necesidades de la industria. Se puede aceptar que la exposición a la luz eléctrica durante la noche altera el funcionamiento y el comportamiento del ser humano, debido a la disrupción del ritmo circadiano en la regulación de las funciones homeostásicas de muchos organismos ${ }^{(6,7)}$. El ritmo circadiano se desarrolla en periodos de 24 horas con manifestaciones de cambios fisiológicos y de comportamiento, relacionados preferencialmente con la información producida por la luz. Se conoce, sin lugar a dudas, que la disrupción del ritmo circadiano por exposición a la luz eléctrica durante la noche produce profundas repercusiones fisiológicas ${ }^{(4,5)}$.

Estudios epidemiológicos sugieren que los trabajadores de los turnos nocturnos expuestos a la luz eléctrica, durante periodos prologados, presentan mayor riesgo de desarrollar cáncer, trastornos del sueño y desórdenes del comportamiento. Además, el exceso crónico de exposición a la luz en la no- che produce un incremento en la incidencia de obesidad y de trastornos metabólicos ${ }^{(6,7)}$. Durante más de 3 billones de años, la vida en la Tierra ha girado alrededor de las condiciones ambientales producidas por la luz ${ }^{(6)}$. Esto ha producido que los organismos unicelulares, las cianobacterias, las moscas de las frutas y los humanos hayan desarrollado un sistema endógeno que sincroniza procesos fisiológicos y de comportamiento bajo la influencia de los ciclos solares externos ${ }^{(6,7)}$. Los relojes biológicos circadianos hacen posible la sincronización de la interacción entre los procesos internos fisiológicos y el medio ambiente externo, permitiendo la identificación del periodo del ciclo en animales en el que no es oportuna la predación y el periodo de procrear. Los ritmos circadianos son definidos como oscilaciones internas que presentan varias características: 1) el periodo del ciclo es de 24 horas en la ausencia de factores ambientales ${ }^{(8)}$. 2) los ritmos son protegidos contra cambios ambientales, como las fluctuaciones de temperatura, etc. ${ }^{(9)}$ y 3) el ciclo puede alternar bajo la influencia de ciertos factores específicos ${ }^{(10)}$.

Un ejemplo común de ritmo circadiano en los mamíferos es la presencia de la alternancia de los estados sueño-vigilia; en las especies diurnas como los humanos, el sueño ocurre durante el periodo de oscuridad, en cambio los animales nocturnos permanecen despiertos durante ciertos periodos del día. El sueño y la vigilia se caracterizan por variaciones hormonales específicas. Por ejemplo, el cortisol se secreta con mayor intensidad temprano en la mañana, precisamente antes de despertar y desciende durante el resto del día, alcanzando sus niveles más bajos al iniciarse el periodo de sueño. El ritmo circadiano influencia varias funciones fisiológicas; el hecho de que se presenten serias condiciones patológicas con la disrupción de estos ciclos demuestra su importancia, tanto en humanos como en mamíferos ${ }^{(6)}$. Las alteraciones del ritmo circadiano originan una serie grande de disfunciones en humanos y otros mamíferos, tales como trastornos cognoscitivos, de comportamiento y un incremento en las enfermedades cardiometabólicas $^{(11,12)}$.

El núcleo supraquiasmático es el director de la orquesta del ritmo circadiano de los mamíferos. Esta estructura está localizada en el hipotálamo anterior directamente por encima del quiasma óptico y está compuesta de aproximadamente 
50.000 neuronas en los humanos y de 8.000 a 20.000 en roedores $^{(13,14)}$.

\section{Mecanismo molecular del ritmo circadiano}

En mamíferos, el reloj molecular del ciclo circadiano está conformado por una asa de retroalimentación autorregulatoria de la trascripción/traducción (para mayores detalles véanse las referencias $\left.{ }^{(6,7)}\right)$.

\section{Relojes circadianos adicionales}

Estos existen en la mayoría de los tejidos de los organismos multicelulares. El sistema nervioso central sirve como el máximo director de este organizado sistema. Los tejidos tienen maquinaria suficiente para poder desempeñar sus funciones. Estos relojes periféricos están bajo el comando del SNC por medio de señales neurales y hormonales ${ }^{(6,7,15,16,17,18,19)}$.

\section{Luz en la noche y el ritmo circadiano}

En contraste con la presencia sostenida de la oscuridad, la exposición crónica a la luz brillante altera la ritmicidad y aplana el ritmo circadiano de los glucocorticoides y modifica la temperatura corporal, dos expresiones principales del ritmo circadiano.

Wright y colaboradores ${ }^{(20)}$ demostraron que individuos expuestos a la luz natural estaban más sincronizados con la luz solar. La exposición a la luz natural redujo la variabilidad en la concentración de melatonina y del ritmo del sueño ${ }^{(20)}$.

\section{El ritmo circadiano regula el metabolismo y viceversa}

Entre los genes rítmicos o cíclicos identificados, muchos pueden tener un papel específico en la coordinación del metabolismo de los nutrientes ${ }^{(21)}$. Por ejemplo, los transportadores de glucosa y los receptores del glucagón, como también varias enzimas comprometidas en el metabolismo de los azúcares y en la biosíntesis del colesterol, se expresan rítmicamente ${ }^{(6,7)}$. Las hormonas relacionadas con los anteriores procesos, tales como el glucagón, la insulina, los corticosteroides y otras, se producen en forma rítmica ${ }^{(6,7)}$. Se han informado fluctuaciones circádicas en las sensaciones del hambre y el apetito ${ }^{(6)}$.

\section{El metabolismo y el reloj circadiano están relacionados}

La relación entre el sistema circadiano y el metabolismo parece ser bidireccional. Así como la disposición del ritmo circadiano causa obesidad, ciertas anormalidades metabólicas alteran el ciclo circadiano, por ejemplo, en humanos, ciertas enfermedades como la obesidad y la anorexia nerviosa, enti- dades relacionadas metabólicamente, presentan alteraciones del ritmo de secreción de ciertas hormonas y de la temperatura corporal(22). En los obesos faltan los ritmos típicos en la captación de la glucosa por ciertos tejidos y la sensibilidad normal a la acción de la insulina ${ }^{(2,3)}$. En los animales experimentales el metabolismo puede afectar el sistema circadiano, tal como se demuestra en la referencia ${ }^{(6)}$.

Hoy en día se reconocen como factores críticos en la adquisición de la energía, el almacenamiento, el consumo y el tiempo en el cual se ingieren los alimentos. Los individuos que consumen más alimentos después de las 8 pm tienen tendencia a presentar mayores índices de acumulación de grasa ${ }^{(24,25)}$. Se ha demostrado que la pérdida de peso es mayor en los que siguen dieta, practican ejercicio y consumen alimentos más temprano y al final del día que los que consumen alrededor de la 8 pm. ${ }^{(26)}$. Existe evidencia de que la ingestión de alimentos en la noche reduce la oxidación de la grasa y aumenta las lipoproteínas de baja densidad ${ }^{(26)}$. El insomnio predispone a la obesidad, al aumento progresivo de peso con el tiempo ${ }^{(27)}$ y a mayor proporción de grasa en el tejido adiposo. Estos hechos indican que cambios en la homeostasis metabólica en los diferentes horarios del día pueden producir regulación variable del peso. Los individuos con el síndrome de ingestión exagerada de alimento en la noche, caracterizada por hiperfagia y necesidad impulsiva de despertar en la noche para consumir alimentos, muestran alteraciones en las hormonas relacionadas con el metabolismo y presentan tendencia a la obesidad ${ }^{(6,27)}$.

\section{Exposición a la luz en la noche y la obesidad}

Debido a que se ha establecido el papel de la luz en la modulación del ritmo circadiano y de ciertas funciones metabólicas, no es extraño que la sobrexposición a la luz artificial afecte el metabolismo. El aumento en la iluminación en la noche se acompaña de obesidad y del síndrome metabólico (figura 1).

\section{Evidencias en animales no humanos}

Las evidencias en animales no humanos de la relación entre la disrupción del ritmo circadiano y la obesidad son amplias y bien estudiadas, sobre las cuales no nos detendremos por brevedad, pero pueden revisarse en las referencias ${ }^{(6,7)}$ (tabla 1).

\section{Evidencia en humanos}

La exposición a >180 lux afecta el ritmo circadiano, pero intensidades menores de luz pueden afectarlo y suprimir la concentración de melatonina ${ }^{(6)}$. Como dato importante, fases de 320 lux son las mínimas requeridas en oficinas y hospitales para evitar accidentes industriales ${ }^{(6)}$. Esto indica que los trabajadores de la noche típicamente están expuestos a niveles de luz más altos que los necesarios para afectar el ritmo circadiano. 
Varios estudios epidemiológicos con trabajadores nocturnos han demostrado que están sometidos a intensidades de luz nocturna capaces de producir trastornos metabólicos ${ }^{(27)}$. Los trabajadores de la salud que hacen turnos nocturnos presentan mayores riesgos de sufrir síndrome metabólico (caracterizado por obesidad, hipertensión e hipertrigliceridemia,

Figura 1. Vías a través de las cuales la exposición a la luz nocturna afecta el metabolismo. Esta exposición puede interrumpir las acciones de los glucocorticoides y de la melatonina, trastorna el sueño y atenúa la expresión genética del ritmo circadiano

\section{Exposición a luz nocturna}

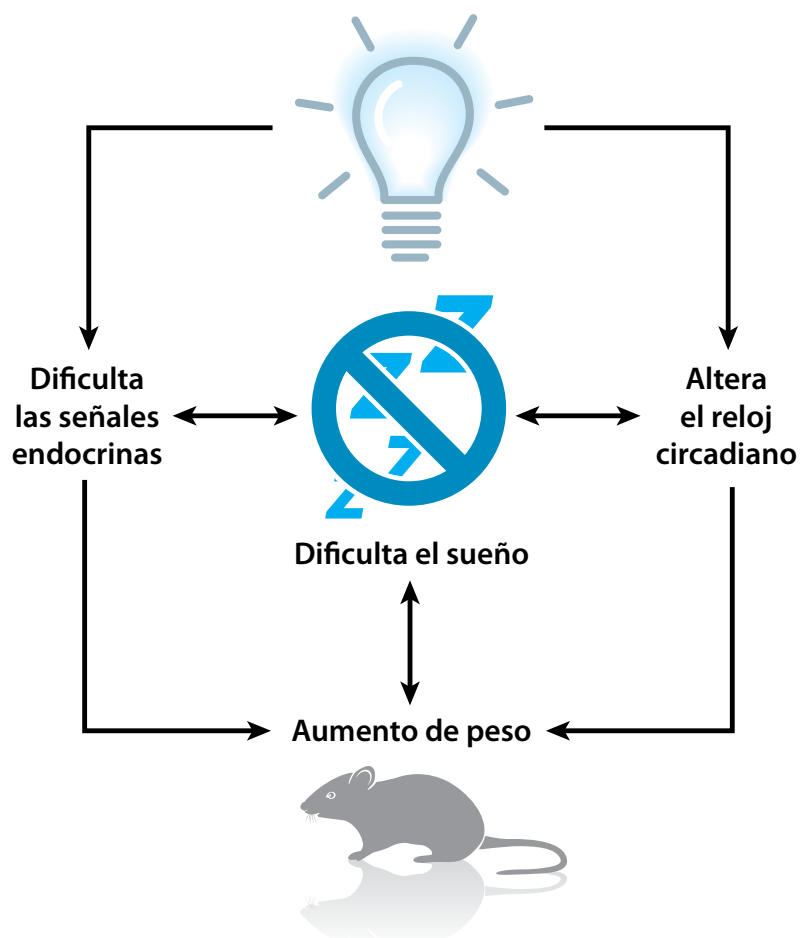

observados especialmente en humanos) que los que trabajan de día ${ }^{(28)}$. Mientras más tiempo se trabaje de noche expuesto a la luz artificial existen mayores probabilidades de presentar estas complicaciones. Esto sugiere que la exposición crónica a la luz artificial puede afectar el índice de masa corporal en mayor proporción que otros predictores de la masa corporal(29-32).

Existen varias limitaciones para obtener conclusiones definitivas acerca de la exposición a la luz nocturna y los trastornos del metabolismo. Primero, los trabajadores nocturnos no solamente están expuestos a la luz artificial sino a otros factores que dificultan sacar conclusiones sin lugar a dudas ${ }^{(6)}$. Segundo, los niveles de luz a los que están expuestos los trabajadores nocturnos no son representativos de la población general. En industrias avanzadas, aproximadamente el 20\% de los trabajadores labora en la noche ${ }^{(33)}$. Además, la exposición a la luz nocturna de los trabajadores que laboran en la noche ocurre también fuera de su trabajo. Como dato importante, la asociación de obesidad con exposición a la luz artificial en la noche ocurre además por fuera de los trabajos industriales. Personas expuestas a niveles de luz nocturna mayores de 3 lux presentan más frecuentemente obesidad, aumento del índice de masa corporal, incremento de la circunferencia de la cadera y aumento de los triglicéridos ${ }^{(6)}$.

La exposición a la luz en la noche puede alterar el ciclo del sueño y de su calidad ${ }^{(6)}$. La deficiencia de sueño puede producir trastornos metabólicos (figura 1).

Otro argumento a favor de la asociación entre la exposición a la luz nocturna y la obesidad está dado por la población amish, que no utiliza la luz eléctrica en ningún momento, ni los aparatos alimentados por esta fuerte energía, televisores, computadores, etc. La prevalencia de obesidad en este grupo es inferior a la de la población general, hecho que no puede explicarse enteramente por la falta de exposición a la luz nocturna, sino que puede deberse, en parte, a aumento de la acti-

Tabla 1. Disrupciones del ritmo circadiano y el metabolismo en animales expuestos a la luz nocturna

\begin{tabular}{l|l|l}
\hline \multicolumn{1}{c|}{ Especie } & \multicolumn{1}{c}{ Tejido } & \multicolumn{1}{c}{ Hallazgos principales } \\
C57BL/6J & Todos los tejidos & Pérdida del ritmo de insulina, formación ectópica de grasa \\
\hline C57BL/6y129 & Hígado & Regulación anormal de la glucosa \\
\hline C57BL/6J & Adipocitos & Propensión a obesidad, ritmos anormales de alimentación \\
\hline C57BL/6J & SNC & Alteración de los periodos de alimentación \\
\hline ICR & Todos los tejidos & Disminución de la grasa, deficiencia en la absorción de la grasa \\
\hline BALB/cyC57BL/6J & Todos los tejidos & $\begin{array}{l}\text { Propensión a la obesidad aumento de la ingestión alimenticia en el día trastornos de } \\
\text { las señales endocrinas }\end{array}$ \\
\hline DBA & Hígado y músculos & Alteración en el metabolismo de la glucosa \\
\hline C57BL/6J & Todos los tejidos & Propensión a la obesidad, hiperinsulinismo, trastornos en los depósitos de grasa \\
\hline C57BL/6 & Todos los tejidos & $\begin{array}{l}\text { Disminución de la masa corporal, alteración de la alimentación, aumento de la } \\
\text { sensibilidad a la glucosa }\end{array}$ \\
\hline C57BL/6 & Todos los tejidos & Alteración de las señales endocrinas \\
\hline
\end{tabular}


vidad física y el control dietario. Como dato digno de mencionar, los amish presentan muchísimo menos cáncer de mama y próstata que la población general de Estados Unidos.

\section{Otros factores responsables de la afectación del metabolismo por la luz}

\section{Melatonina}

La melatonina es una molécula endógena secretada por la pineal durante la noche, tanto en animales nocturnos como diurnos $^{(34)}$. La secreción de esta hormona es potencialmente inhibida por la exposición a suficientes niveles de luz y duración durante la noche, en roedores y humanos. Si se exponen las personas durante horas a 45 lux durante la noche, la melatonina disminuye el $60 \%{ }^{(35)}$.

La melatonina ha sido ampliamente investigada en roedores $^{(6)}$, pero no en humanos. Se ha propuesto que la melatonina podría ser de utilidad en la terapia de obesidad, pero las evidencias de esta propuesta son mínimas y no convincentes. Se ha sugerido que niveles bajos de melatonina en la noche predisponen a la presentación de diabetes. La administración de melatonina en humanos estimula la producción de glucagón, aumentando la secreción de insulina, pero en ratas la melatonina disminuye la secreción de insulina.

Aunque existen evidencias de que la supresión de la melatonina puede contribuir al aumento de peso, algunas experiencias sugieren que la melatonina no es el factor primario a través del cual la luz afecta el metabolismo ${ }^{(36)}$. Se ha propuesto que la melatonina sería efectiva en la terapia antiobesidad ${ }^{(37,38)}$; sin embargo, existe muy poca investigación sobre la eficacia de la melatonina en la terapia de la obesidad en humanos. Un estudio informó de la asociación de la melatonina con el índice de masa corporal en mujeres, pero no en personas con peso normal, ni en hombres ${ }^{(39)}$. La disminución de la secreción de melatonina se asocia al riesgo de presentar diabetes tipo 2 .

Aunque hay evidencia de que la supresión de la secreción de melatonina puede contribuir al aumento de peso, existen dudas de si la melatonina en realidad es el factor más importante a través del cual la luz produciría los efectos nocivos sobre el metabolismo: 1) la supresión de la secreción de la melatonina requiere alta intensidad de iluminación nocturna (45 lux ${ }^{(34)}$, una mayor intensidad que la descrita en el ambiente normal de sueño. 2) Aun con la exposición nocturna a niveles de luz por debajo de la necesaria para suprimir la secreción de la melatonina se observaron cambios metabólicos, 3) Varias cepas de roedores que no presentan secreción pineal de melatonina demostraron cambios del metabolismo con la exposición a la luz en la noche ${ }^{(6)}$.

\section{Glucocorticoides y el metabolismo}

Por falta de espacio, remitimos a los lectores sobre estos tópicos a la referencia número 6 , donde se darán detalles, exis- ten evidencias de que la alteración de los glucocorticoides no es crítica para los cambios observados en el metabolismo que sí presentan con la exposición a la iluminación nocturna ${ }^{(6)}$.

\section{El sueño y el metabolismo}

La exposición a la luz puede alterar el metabolismo por disrupción del sueño. Trastornos del sueño pueden afectar profundamente el metabolismo y contribuir a múltiples patologías, incluyendo el síndrome metabólico ${ }^{(6)}$. La disminución del sueño en humanos está asociada a riesgos altos de acompañarse de obesidad, en todas las edades; tan solo cinco días de sueño insuficiente pueden causar el exceso de ingestión de energía, especialmente en la tarde, con incremento del peso ${ }^{(6)}$.

La asociación de la supresión de la melatonina, la disrupción del ritmo circadiano, cambios en el eje hipotálamo-hipófisis adrenal y trastornos del sueño, todos pueden contribuir a los efectos deletéreos que se presentan con la exposición a la luz en la noche. Considerados todos estos factores conjuntamente, parece que los efectos negativos que produce la exposición a la luz en la noche se deben primordialmente a los efectos metabólicos originados en la disrupción del ritmo circadiano ${ }^{(6)}$.

\section{Posibles medidas preventivas}

Cerca del 99\% de la población de Estados Unidos y Europa está sobreexpuesta a la luz urbana y muchas personas utilizan luz eléctrica en la noche, en la televisión, radios, computadores, etc. El 20\% de la población está expuesto a altos y prolongados niveles de luz eléctrica ${ }^{(6)}$. No sólo los humanos sino también las plantas y varias especies de animales pueden ser afectados con la exposición a la luz nocturna. El uso crónico de la iluminación nocturna afecta la reproducción, el comportamiento, la predación y la migración de varias especies ${ }^{(6)}$. Sin embargo, no toda la iluminación nocturna afecta igualmente el ritmo circadiano. Las células fotosensibles de los ganglios retinales que proyectan la luz al sistema nervioso responden más a la región azul del espectro visible (entre 450-485 nm); longitudes de onda de iluminación más largas influencian poco el ritmo circadiano. Con manipulación de la longitud de onda de la luz se pueden bloquear los cambios fisiológicos inducidos por la luz. Está en experimentación la prevención de la exposición a la luz azul con utilización de gafas especiales. Con el uso de gafas que excluyan las longitudes de onda de la luz por debajo de $520 \mathrm{~nm}$ se puede evitar la supresión de la secreción de la melatonina en trabajadores nocturnos ${ }^{(6)}$. Con la utilización de pulsos de luz durante la noche y el uso de la gafas oscuras en la mañana se puede conseguir aumento de la ciclos del sueño en la casa ${ }^{(6,40)}$.

\section{Conclusiones}

La exposición a la luz nocturna puede alterar los mecanismos utilizados en el ritmo circadiano, no sólo a nivel del sistema nervioso central sino también en los tejidos periféricos. 
La disrupción del funcionamiento del ritmo circadiano está asociada a la presencia de obesidad en animales y humanos. Además, la iluminación crónica nocturna produce cambios en el horario de la ingestión de alimentos con incremento de los depósitos de grasa. Existe evidencia de que el aumento global en la prevalencia de la obesidad y de los trastornos metabólicos coinciden con el aumento de la iluminación nocturna y el cambio en los turnos de trabajo. Está claro que la disrupción del ritmo circadiano por la exposición a la iluminación nocturna trastorna el metabolismo. Sin embargo, debe tenerse en cuenta que las alteraciones del ritmo circadiano producidas por otras causas pueden producir efectos metabólicos similares, lo que puede en ciertos casos hacer difícil establecer cuál es el principal factor determinante. Algunos investigadores dudan de los efectos nocivos de la exposición aguda y crónica a la luz nocturna sobre el metabolismo, pero esta breve revisión apoya las evidencias de que la iluminación nocturna trae nocivas consecuencias para el sistema circadiano y que coadyuva en la presentación del síndrome metabólico y sus componentes, hipertensión, diabetes, obesidad y predisposición a las enfermedades cardiovasculares.

Se necesitan investigaciones futuras para definir a través de qué mecanismos la exposición a la luz en la noche modifica los genes del ritmo circadiano y qué componentes necesitan modificarse o alterarse para producir los efectos metabólicos descritos. Adicionalmente, existen muy pocos estudios clínicos que demuestran los efectos de la exposición a la luz durante la noche.

Investigaciones futuras deben estudiar qué niveles de luz en la noche son los menos nocivos en los hogares, ancianatos, hospitales, etc., donde los individuos pueden ser más susceptibles a la exposición a la luz eléctrica.

Es necesario definir, sin lugar a dudas, los efectos de la exposición crónica a luz nocturna sobre la salud de los seres humanos y sus consecuencias, lo que ayudaría a determinar las dosis de iluminación nocturna dentro y fuera de los hogares, que eviten efectos negativos debidos a esta fuente de energía.

\section{Referencias}

1. Caballero B. The global epidemic of obesity: an overview. Epidemiol Rev. 2007; 29:1-5.

2. Symonds ME, Sebert B, Budge H, The obesity epidemic from the environment epigenetics- no simply a response to direct manipulation in a thermoneutral environment. Front Genet. 2001; 2:24.

3. National Academy of Engineering. Greastest engineering achievements of the 20th century. Engineering. 2000. Htt:/www.greatest achievement.org/.

4. Fonken LK, Nelson RJ. Iluminating the deleterious effect of light at night. F100 Med Rep. 2001;13-18.

5. Navarra KJ, Nelson RJ. The dark side of light at night: phsiological, epidemiological, and ecological consequences. J Pineal Res. 2007; 43(3): 215-224.

6. Laura K, Fonken and Randy J Nelson. The effects of light at night on circadian clocks and metabolism. Endocrine Rev. 214; 35:648-670.

7. Gerhart- Hinces Z, Lazan A M. Circadian metabolism in the light evolution. Endocrine Rev. 2015; 36:289-304.

8. Ashoff J. Circadian rhythms in man.Science. 1965; 148 (3676): 1427-1432.

9. Ruby NF, Burns DE, Hellen HC. Circadians rhythms in the suprachiasmatic nucleus are temerature-copensated and phase- shited by heat pulses in vitro. J Neurosci. 1999; 19 (9): 8630-8636.

10. Ralph MR, Menaker M. A mutation of circadian system in golden hamsters. Science. 1988; 241 (4870): 1225-1227.

11. Takahashi JS, Hong HK, Ko CH, et al. The genetics of mammalian circadian order and disorder: implications for physiology and disease. Nat Rev Genet. 2008; 9 (10): 764-775.

12. Zee PC, Attarian H, Videnovic A. Circadian rhytm abnormalities. Continuum. 2013; 191 (sleep disorders): 132-147.

13. Van den Pol AN. The hypothalamic suprachiasmatic nucleus of rat: intrinsic anatomy. J Comp Neurol. 1980; 191 (14) : 661-702.

14. Lydic R, Albers HE, Tepper B, et al. Three dimensional structure of the mammalian suprachiasmatic nuclei: a comparative study of five species.J Comp Neurol. 1982; 201 (3): 225-237.

15. Lydic R, Schoene Wc, Czeisler CA, et al. Suprachiasmatic region of the human hypothalamus: homolog to the primate circadian pacemaker? Sleep. 1980; 2 (3): 355-361.

16. Rusak B, Groos G. Suprachiasmatic stimulation phase shifts rodents circadian rhytms. Science. 1982;215 (4538): 1407-1409.

17. Reddy AB, Maywood ES, Karp NA, et al. Glucocorticoid signaling synchronizes the liver circadian transcriptome. Hepatology. 2001; 45 (6): 1478-1488.

18. McNamara P, Seo SB, Rudic RD, e al. Regulation of CLOCK and MOP4 by nuclear hormone receptores in the vasculature: a humoral mechanism to reset a periphenal clock. Cell. 2001;105(7): 877-889.

19. Guo H, Brewer JM, Champhekar A, et al. Differential control of preripheral circadian rhytm by suprachiasmati-depend neural signals. Proc Natl Acad Sci USA. 2005;102 (8): 3111-3116.

20. Wright KP Jr, McHill Aw Birks BR, et al. Entrainment of the human circadian clock to the natural light-dark cycle. Curr Biol. 2013; 23 (16): 1554-1558.

21. Eckel-Maham KL, Sassone-Corsi P, et al. Coordination of the transcriptome and metabolome by the circadian clock. Proc Natl Acad Sci USA. 2012; 109(14): 5541-5546.

22. Scheer FA, Morris CJ, Shea SA. The internal, circadian clock increases hunger and apetite in the evening independent of food intake and other behaviors. Obesity. 2013; 21 (3): 421-423.

23. Lee A, Ader M, Bay GA, et al. Diurnal variation in glucose tolerance. Ciclic suppression of insulin action and insulin secretion in normal- weight, but no obese subjects. Diabetes. 1992;41(6):750-79.

24. Baron KG, Reid KJ, Kern AS, et al. Role of sleep timing in caloric intake and BMI. Obesity. 2011;119 (7): 1374-1381.

25. Baron KG, Reid KJ, Kern AS, et al. Contribution of evening macronutrients intake to total caloric intake and body mass index. Apetite. 2013;60(1): 246251.

26. Jakubowicz D, Barnes M, Wainstein J, et al. High caloric intake at breakfast versus dinner differentialty influences weigth loss of overweight and obese women. Obesity (Silver spring). 2013;21 (12): 2504-2512.

27. Wang XS, Armstrong ME, Cairns BJ, et al. Shift Work and chronic disease: The epidemiologcal evidence. Occups Med. 2010;67(1):54-57.

28. Pietroiusti A, Nery A, Soma G, et al. Incidence of metabolic síndrome among night-shift healthecare Workers. Occups Environ Med. 2010;67(1):54-57.

29 Sookian S, Gemma G, Fernandez Gianotti, et al. Effects of rotating shift work on biomarkers of metabolic síndrome and inflamation. J Intern M. 2007;261 (3): 285-292.

30. Esquirol Y, Bongard V, Mabile L, et al. Shift work and metabolic sydromre: respective impacts of job strain, thypical activity, and dietary rithms. Chronobiol Int. 2009; 26 (3) 544-559.

31. De Bacquer D, Van Risseghem M,et al. Rotating shift wok and th metabolic síndrome; a prospective study. Int J epidemol. 2009;26(3): 544-559.

32. Lin YC, Hsiao TJ, Chen PC. Persistent rotating shift work exposure accelarates development to metabolic sydrome among middle-aged females employees: a fice years follow up. Cronobiol Ind. 2009;26 (4): 740-755.

33. Monk TH. Shift Work. In: Kryger MH, Roth T, Dement WC, eds. Principles and practice of Sleep Medicine. Philaldephia, PA: WB Saunders; 2000:471-476

34. Reiter RJ. Melatonin: The chemical expresión of darkness. Mol Cell Endocrinol. 191;791 (1-3): C153-C158.

35. Brainard GC, Lewy Aj, Menaker M, et al. Dose-response relationship between light irradiance and the suppression teens. Brain Res. 1988; 454 (1-2): 212218.

36. Brainard GC, Rollag MD, Hanifin JP. Photic regulaton of melatonin in human: ocular and neural signal transduction. J Biol Rhytsms. 1997;12(6): 537-546.

37. Nduhirabandi F, du Toit EF, Lochner A. Melatonin and the metabolic sydrome: a tool for effective therapy in obesity associated abnormalities? Acta Physiol. 2012; 205(2): 209-233.

38. Reiter RJ, Tan DX, Korkamaz A, MaS. Obesity and metabolic síndrome: association with chronodisruption, sleep deprivation and melatonin suppression. Ann Med. 2012; 44(6) 564-577.

39. McMullan CJ, Schernhammer ES, Rimm ES,et al. Melatonin secretion and the indcende $\mathrm{f}$ thype 2 diabetes. JAMA, 2013;309 (13):1388-1396.

40. Kayumov L, Casper RF, Hawa RJ, et al. Blocking low wavelenght light prevents nocturnal melatonin suppresion with no adverse effect on perfomance during simulated shift work. J Clin Endocrinl Metab. 2005;90(5): 2755-2761. 